\title{
ERRATA
}

\section{Propagation of Magneto-elastic Waves in a Perfectly Conducting Plate Extending to Infinity in Vacuum}

\author{
By C. M. Purushothama ${ }^{1}$ )
}

The author regrets that some corrections are to be made in the results reported in lis recent paper with the above title $\left.[1]^{2}\right)$.

In the second of equations (14) the exponential factor $e^{q T}$ does not occur so that

$$
\delta_{3}=\frac{k^{2} V^{2}}{q c^{2}} \kappa_{0} H^{2}
$$

The dispersion equations for symmetric and antisymmetric cases given by (15a, b) can be written as

$$
\begin{aligned}
& \frac{\tanh \left(1-\frac{V^{2}}{\alpha^{2}+V_{A}^{2}}\right)^{1 / 2} k T}{\tanh \left(1-\frac{V^{2}}{\beta^{2}}\right)^{1 / 2} k T} \\
& =\frac{\left(2-\frac{V^{2}}{\beta^{2}}\right)\left\{2-\frac{V^{2}}{\beta^{2}}-\frac{V_{A}^{2} V^{2}}{\beta^{2} C^{2}}\left(1-\frac{V^{2}}{\alpha^{2}+V_{A}^{2}}\right)^{1 / 2}\left(1-\frac{V^{2}}{c^{2}}\right)^{-1 / 2} \tanh \left(1-\frac{V^{2}}{\alpha^{2}+V_{A}^{2}}\right)^{1 / 2} k T\right\}}{4\left(1-\frac{V^{2}}{\alpha^{2}+V_{A}^{2}}\right)^{1 / 2}\left(1-\frac{V^{2}}{\beta^{2}}\right)^{1 / 2}\left\{1-\frac{V_{A}^{2} V^{2}}{2 \beta^{2} c^{2}}\left(1-\frac{V^{2}}{\alpha^{2}+V_{A}^{2}}\right)^{1 / 2}\left(1-\frac{V^{2}}{\beta^{2}}\right)^{1 / 2} \tanh \left(1-\frac{V^{2}}{\beta^{2}}\right)^{1 / 2} k T\right\}}, \\
& \frac{\tanh \left(1-\frac{V^{2}}{x^{2}+V_{A}^{2}}\right)^{1 / 2} k T}{\tanh \left(1-\frac{V^{2}}{\beta^{2}}\right)^{1 / 2} k T} \\
& =\frac{4\left(1-\frac{V^{2}}{\alpha^{2}+V_{A}^{2}}\right)^{1 / 2}\left(1-\frac{V^{2}}{\beta^{2}}\right)^{1 / 2}\left\{1-\frac{V_{A}^{2} V^{2}}{\beta^{2} c^{2}}\left(1-\frac{V^{2}}{\alpha^{2}+V_{A}^{2}}\right)^{1 / 2}\left(1-\frac{V^{2}}{\beta^{2}}\right)^{1 / 2} \operatorname{coth}\left(1-\frac{V^{2}}{\beta^{2}}\right)^{1 / 2} k T\right\}}{\left(2-\frac{V^{2}}{\beta^{2}}\right)\left\{2-\frac{V^{2}}{\beta^{2}}-\frac{V_{A}^{2} V^{2}}{2 \beta^{2} c^{2}}\left(1-\frac{V^{2}}{\alpha^{2}+V_{A}^{2}}\right)^{1 / 2}\left(1-\frac{V^{2}}{c^{2}}\right)^{-1 / 2} \operatorname{coth}\left(1-\frac{V^{2}}{\alpha^{2}+V_{A}^{2}}\right)^{1 / 2} k T\right\}} .
\end{aligned}
$$

1) Department of Mathematics, Indian Institute of Technology, Kharagpur, India.

2) Number in brackets refer to Reference, page 254. 
In all cases of acoustic wave propagation, the ratio $V_{A} V / \beta C$ is extremely small and, therefore, the radiation effect is negligible both for long waves and short waves. For symmetric waves, this is evident because of the fact that $\tanh \theta$ is finite and lies between 0 and 1 for all values of $\theta$; for antisymmetric waves the approximation can be seen to be justified after cross multiplication. It follows that the equations (16a) and (16b) hold without any restriction.

In equation (20) $\kappa$ should replace $\kappa_{0}$. In the third term in equation (21) and in $\sigma_{3}, \kappa_{0}$ is to be changed to $\kappa^{2} / \kappa_{0}$, and they should be read without the factor $e^{q T}$.

Noting that $\sigma_{1} a=\sigma_{2}$, equations (22) and (23) reduce, respectively, to

and

$$
e^{4 i k b T}=\left(\frac{\sigma_{3}+i b \sigma_{1}}{\sigma_{3}-i b \sigma_{1}}\right)^{2}
$$

$$
B_{3}=A_{3} e^{2 i k b T} \frac{\sigma_{1} b+i \sigma_{3}}{\sigma_{1} b-i \sigma_{3}},
$$

so that $B_{3}= \pm A_{3}$ for all $\varphi$ depending upon the mode, and (26) simplifies to $B_{3}$ $=(-1)^{m-1} A_{3}$.

\section{ReFEREnCE}

[1] C. M. Purushothama, Pure and Appl. Geophysics 61 (1965/II), 60.

(Received 15th February 1966) 